made by the manufacturers and retailers, the difference should be overwhelming. But the item of palatability is something to be considered, and the slight from the adjacent tissues, partly by cutting and partly difference in the percentage of starch might well be by tearing. The index finger introduced into the waived in favor of the great difference in taste.

There is another and more serious side to this question of diabetic foods. That they are in the highest degree fraudulent has, I consider, been proved. They are in addition a positive danger, for the diabetic accepting as truth the assertions that they are nonstarchy, takes into his system that which even the circulars of the manufacturers admit to be virtually a poison in its effect on the course of the disease, and thus innocently more than counteracts the benefit which he otherwise would derive from his medical advise1. In conclusion, I have to express the hope that every practitioner will do his utmost to discourage the use of these fraudulent and dangerous foods, and to drive them out of the market.

A CASE OF MACEWEN'S OPERATION FOR THE RADICAL CURE OF INGUINAL HERNIA IN A WOMAN SIXTY-THREE YEARS OF AGE. ${ }^{1}$

BY G. H. MoNks, M.D.,
Surgeon to the Boston Dispensary i. Surgeon to Out-Patients at the
C'arney Hospital.

The patient was admitted to the Carney Hospital on October 1, 1887, at a time when I was temporarily in charge of the surgical wards. She was small in stature, but well nourished and in remarkable good physical condition for one of her years. She could not remember that she had ever been sick in bed before.

She attributed the hernia from which she suffered to a fall about six years previously, shortly after which she experienced occasional "sickening pains" in the lower part of the abdomen, on the left side. The left labium became gradually enlarged, and there was pain in that region more or less constant, particularly severe during defecation. The patient had been advised to wear a truss, but she stated that with the use of one she had never been able to satisfactorily control the rupture. Eventually, the swelling became so large and troublesome that she was unable to get about. Being at this time in no condition to help herself, and becoming more and more a burden to her family and friends, she came to the Hospital, fully resolved to have anything done which offered a chance of cure.

An examination showed the existence of an inguinal hernia on the left side, of the size and shape of a large pear, the body occupying the left labium, and the neck extending to the external ring. The ring was so large that three fingers could be inserted without difficulty. The hernia was readily reducible.

Dr. A. T. Cabot saw the case in consultation, and, under the circumstances, advised operation. On October 12th, after the patient had been properly prepared, ether was given and the operation was carried out with thorough antiseptic precautions, Drs. Whitman and Monro kindly assisting.

The parts were shaved and rendered aseptic by washing with ether, and then with corrosive sublimate solution, with soap. A straight incision was made from the region of the ring to the lower extremity of the hernial protrusion, and this was deepened until

I Read before the Suffolk District Medical Society, Surgical Secthon, January $4,1 \% 88$. the sac was reached. The sac, which was unusually adherent, was then separated as carefully as possible by tearing. The index finger

It was then possible to separate with the finger-tip the peritoneum from the abdominal margins of the internal ring. The sac was now puckered up by a continuous suture of stout chromicized catgut, in the manner recommended by MacEwen, and the needle was pushed through the abdominal wall from behind forwards. On drawing upon this suture, the puckered sac was with the assistance of the finger made to occupy a position within the abdomen, where it considerably overlapped the margins of the inner ring. The suture, thus drawn tight, was made fast in the muscles of the abdomen. 'The walls of the canal were now drawn together with chromicized gut, so that the conjoined tendon and Poupart's ligament should be approximated as closely as possible. It was now evident that it would be impossible, on account of the size of the cavity left after removal of the sac, to sew up the wound in the ordinary way without leaving pockets. A large number of sutures of fine catgut were, therefore, buried, and the margins of the wound brought together with a continuous silk suture. No drainage was used. Iodoform was sprinkled on the wound, and flexible collodion, layer after layer, with strips of cotton. This was covered with a large pad of absorbent cotton kept in place by a firm $\mathrm{T}$. bandage.

The patient rallied from the immediate effects of the operation, but the recovery was complicated by a failure of the wound to unite in that part superficial to the external ring. It is difficult to say whether this was due to some defect in the antiseptic precautions or to the age of the patient, which was certainly unfavorable for rapid repair. The deep sutures apparently held the walls of the inguinal canal in perfect apposition. The condition of the parts gradually improved by the free use of antiseptic solutions, and the wound slowly healed by granulation. The patient now sits up a few hours each day.

There has never been any sign of a recurrence, though no bandage or pad of any kind has been used since the first few days succefding the operation.

I report this case as interesting on account of the age of the patient, but am well aware that no case should be considered cured until a period of, at least, one year has passed without recurrence, during which time no artificial support of any kind has been worn. It certainly does not seem to be advisable to attempt a radical cure in old persons, except in such cases as insist upon an operation, after they have been made acquainted with the dangers, as well as with the chances of recovery and of a permanent cure.

\section{A CASE OF MACEWEN'S OPERATION FOR THE RADICAL CURE OF CONGENITAL INGUINAL HERNIA IN A BOY. ${ }^{1}$}

BY ROYAL WHITMAN, M.D.

Surgeon to the Orthopsdic Department at the Boston Dispensary.

THE patient, a boy eleven years of age, was brought to me with a left scrotal hernia, which had existed since birth. Various trusses had been applied with ' Read before the Sutfolk District Medical Society, Surgical Section, January 4 th, 1888 . 
but partial relief, and finally their use had been abandoned. Both mother and child were anxious for a radical operation, as the affection prevented the boy from playing with the other children, and was the cause of more or less continual discomfort, with at times severe pain. The hernia was about the size of an orange. The external opening admitted two fingers, the bowel slipping easily in and out of the abdomen cavity.

On May 9, 1886, eight months ago, I performed MacEwen's operation, assisted by Drs. Monks and Conant. The sac of the hernia, congenital, was easily dissected out, divided above the testicle, separated from the cord and returned to the abdominal cavity, the lower segment being sewed together over the testicle to form the tunica vaginalis. The sac was attached to the abdominal wall, and the canal closed with thick chromicized gut. The wound was closed with fine gut, several strands of which being placed superficially for drainage. 'The dressing consisted of iodoform ganze, covered with a thick pad of corrosive sublimate cotton, and held in place by a $\mathrm{T}$ bandage of thin sheet rubber, which provided equable pressure, and prevented any contamination of the dressing. The leg was then flexed upon the body, and securely banduged to a bent wire frame, known as the "Cabot hip-splint." This apparatus was of great service, preventing the child from tearing off the dressing; and I should strongly recommend its use for children, where, as in this case, constant attendance cannot be provided. In any event, too, this immobilization of the leg must materially aid in the cure.

During the night there was retention of urine, and afterwards elevation of temperature, which rose to $103^{\circ}$ on the third day. The superficial stitches were then removed and the wound explored, but nothing was found to account for the fever except some induration about the parts. The temperature then fell to normal. On the twelfth day when the dressing was removed the wound had closed. During the third week the child who is now presented for examination, was about as usual, and has since suffered no discomfort, no bandage or support having been worn.

\section{Ineparts of Sacieties. \\ BOSTON SOCIETY FOR MEDICAL IMPROVE- MENT.}

F. B. Harrington, M.D., SECRETARY.

February 27, 1888, the President, Dr. O. F. WADSWORTH, in the chair.

RESULT OF RESECTION OF THE ANKLE-JOINT.

Dr. CABOt showed eight cases of resection of the ankle-joint illustrating the final result that can be hoped for from this operation. Tracings made by Mr. C. L. Scudder from several of the cases, were also shown; which gave the mobility of the diseased joints as compared with the well ones. The patients had all been treated at the Children's Hospital, and were not selected cases, as all of those operated upon by the reader, whose addresses he knew, had been sent for, and all of those who responded were shown.

The periods elapsed since the operation varied from one to seven years, and the joints were all solidly healed and free from tenderness or swelling. In one large girl operated upon seven years ago, in whom the astragalus and a considerable portion of the lower end of the tibia and fibula with the malleoli, were removed, there was a tendency to pes varus, not sufficient, however, to cause inconvenience. The joint was firm and caused no discomfort, although she was constantly on her feet.

One rather puny boy had a slight degree of pes equinus, but with these exceptions the joints were in good position. In several instances, the bones removed, were shown with the patients. In all of the cases the disease was a caries of the smaller bones, often affecting the ends of the long bones and probably always tuberculous in character. In one case, disease of the knee-joint (white swelling), and in another, caries of the lower dorsal vertebræ complicated the condition. The reader said that this operation was often, perhaps usually, unsatisfactory in adults, but that in children it almost invariably resulted in cure and in the restoration of a useful joint, as the cases present would help to show. He then went on to say : "In regard to the operation, the first important thing is the thorough removal of all diseased tissue, osseous, cartilaginous and synovial. In my earlier cases I of ten tried to limit the operation to the parts evidently diseased, and scraped out the softened portions with a sharp spoon. In dealing with the small bones, however, I soon found that the part left, almost certainly became affected with the disease and had subsequently to be removed, so that I later adopted the plan of removing the whole of any of the small bones which showed unmistakable caries. The only exception to this rule was made in cases of caries of the os calcis, in which the disease seemed to be wholly extra-articular. Of the longer bones the metatarsals were treated by the removal of the diseased ends.

When we come to the tibia and fibula, we have strong reasons for trying to save all of the healthy bone possible:

First: Interference with the epiphysial cartilage checks the future growth of the limb and may unnecessarily add to the shortening.

Second: The malleoli when they can be preserved add greatly to the strength and steadiness of the new joint. The shortened foot falls in between them and they give it much lateral support. It is therefore better, when possible, to thoroughly scrape out the diseased tissue in these bones and not, except in extreme cases, to saw off the ends evenly.

Several of the cases here this evening show, however, that even when the malleoli are lost, a very useful joint may still be obtained. After the removal of the bones the diseased synovial membrane should be carefully dissected out. This brings us to speak of the incision used in these cases. I have always used the old method with two lateral incisions, which avoid the injury of any tendon or vessels, and which $I$ have found to give easy access to all parts of the cavity. The incisions should be of good length, as the thoroughness of the operation is important, and the skin readily heals.

In the after-treatment, one or two points seem worthy of attention: In the dressings, iodoform is a valuable auxiliary, as it seems to have the power of checking any subsequent tubercular formation which might start from small portions of granulation tissue, overlooked in the dissection. A seton of iodoform 